\title{
Inverse melting in lattice-gas models
}

\author{
Santi Prestipind* \\ Università degli Studi di Messina, Dipartimento di Fisica, \\ Contrada Papardo, 98166 Messina, Italy
}

(Dated: November 12, 2018)

\begin{abstract}
Inverse melting is the phenomenon, observed in both Helium isotopes, by which a crystal melts when cooled at constant pressure. I investigate discrete-space analogs of inverse melting by means of two instances of a triangular-lattice-gas system endowed with a soft-core repulsion and a shortranged attraction. To reconstruct the phase diagram, I use both transfer-matrix and Monte Carlo methods, as well as low-temperature series expansions. In one case, a phase behavior reminiscent of Helium emerges, with a loose-packed phase (which is solid-like for low temperatures and liquid-like for high temperatures) extending down to zero temperature for low pressures and the possibility of melting the close-packed solid by isobaric cooling. At variance with previous model studies of inverse melting, the driving mechanism of the present phenomenon is mainly geometrical, related to the larger free-energy cost of a "vacancy" in the loose-packed solid than in the close-packed one.

PACS numbers: 05.20.Jj, 61.20.Ja, 64.10.+h, 64.70.Dv
\end{abstract}

Keywords: Inverse melting; solid-liquid and solid-solid transitions; transfer-matrix method; low-temperature series expansions

*Electronic address: Santi.Prestipino@unime.it 


\section{INTRODUCTION}

Inverse melting (IM) is hardly mentioned in a catalog of Helium oddities. Yet, Helium provides the only example of an elemental solid that can be melted isobarically by lowering the temperature, although this only occurs in a small range of pressures. This striking phenomenon, which is still poorly understood, is evidenced in the low-temperature phase diagrams of both ${ }^{3} \mathrm{He}$ and ${ }^{4} \mathrm{He}$ by a decreasing profile of the solid-liquid coexistence pressure $P_{\text {cox }}$ as a function of temperature $T$ [1]. Another kind of IM is provided by P4MP1 polymer

solutions [2], where a tetragonal crystal undergoes amorphization on cooling, accompanied by heat release.

The existence of IM fights against common sense but by no means disproves thermodynamics [3, 4]. The slope of $P_{\operatorname{cox}}(T)$ is ruled by the Clausius-Clapeyron equation,

$$
\frac{\mathrm{d} P_{\operatorname{cox}}}{\mathrm{d} T}=\frac{S_{2}-S_{1}}{V_{2}-V_{1}}
$$

where $V_{1}, V_{2}$ and $S_{1}, S_{2}$ are the volume and entropy values of the coexisting phases. Nothing prevents a phase transition to occur, at fixed external pressure, from a low-temperature phase 1 to a high-temperature phase 2 with both volume contraction and positive latent heat, hence with negative $\Delta S / \Delta V$ (this is the case, for instance, of ordinary ice and water). What is peculiar to Helium IM is that the more compact and more entropic phase is solid rather than liquid. The case of $\mathrm{P} 4 \mathrm{MP} 1$ is different: Here $\Delta S / \Delta V>0$, but the solid still lies on the high-temperature side of the transition.

Generally speaking, IM requires some microscopic mechanism by which spatially-confined particles can nonetheless have more entropy than the coexisting liquid. In ${ }^{3} \mathrm{He}$, the nuclear spins are more coupled (i.e., less free to orient independently of each other) in the liquid than in the solid phase. Inverse melting of P4MP1 is explained by a larger amount of polymer conformations in the crystal than in the amorphous state, due to a more open crystal structure.

Pursuing the analogy with the above systems, the few models of IM proposed so far have invariably focussed on systems of particles with internal degrees of freedom [5, 6]. When the single-particle states are taken to be more degenerate in the ordered than in the disordered phase, IM can occur. In practice, one needs a very fine tuning of the single-particle spectrum to obtain a realistic IM scenario. 
The purpose of this paper is to show that IM - or an analog of it - is also observed in systems that are hosted on a lattice. The model presented here is a classical lattice-gas system which, with no other ingredients than the radial dependence of its pair-interaction strenght, exhibits a phase behavior that is reminiscent of Helium. In particular, 1) the system can exist in a loose-packed phase down to zero temperature for low enough pressures; and 2) in a range of pressures, the close-packed solid melts into a less dense structure upon cooling. This phase is crystalline at very low temperatures, but can be a liquid at higher temperatures. Besides the similarities, however, the present IM has conceivably little to do with Helium: While IM of the latter has a quantum-mechanical origin, the phenomenon herein discussed heavily relies on the steric constraints being determined by the interplay between lattice geometry and interaction. Specifically, it deals with the different free-energy cost, at low temperature, of vacancy-like excitations in the loose-packed solid and in the close-packed one. In fact, any lattice potential that is provided with an extended hard-core repulsion, a shoulder, and a thin attractive well would prove adequate to bend downward $P_{\text {cox }}(T)$ at $T=0$.

The rest of the paper is organized as follows: In Section 2, after introducing a class of lattice gases that could possibly share some features in common with Helium, I outline an analytic method for checking the existence of IM. Then, in Section 3, I describe the numerical techniques that are used to work out the phase diagram of such a lattice gas. Results are presented in Section 4, where a comparison is made between two similar case studies, in an attempt to unveil general rules of behavior. Some further remarks and conclusions are given in Section 5.

\section{MODEL}

Classical lattice gases provide caricatural descriptions of real fluids that, while broadly preserving the topology of the phase diagram, are by far much simpler to study both analytically and numerically [7, 8, 9, 10]. If the aim is finding a lattice-gas system with a Helium-type phase diagram, but with no ambition to model a real substance, working in two dimensions is by no means restrictive, in fact it is convenient for a twofold reason: The transfer-matrix method, which is the most powerful technique for reconstructing numerically the phase diagram of a lattice gas, is a viable tool only in two dimensions. Moreover, with 
an eye to making use also of low-temperature expansions for the analysis, enumeration of ground-state defects/excitations is much easier in two than in three dimensions.

The type of models here considered is a lattice-gas system hosted on the triangular lattice. A given lattice site can be either occupied (by a single particle) or empty. Calling $c_{i}=0,1$ the occupation number of site $i$, the lattice-gas Hamiltonian reads $\sum_{i<j} v(|i-j|) c_{i} c_{j}$, with a pair potential $v$ depending on site-site separation only. In the very first place, this potential is asked to meet two conditions: 1) It must allow for the existence of two disordered phases, "vapor" and "liquid"; 2) two different crystalline phases should be stable at $T=0$, say "solid A" at high pressures and "solid B" at low pressures. However, the real challenge is to find a model where "solid B" and "liquid" are actually the same phase or, at least, have similar thermodynamic properties along the transition line.

I have shown elsewhere [10] that an extended hard core in the potential, when associated with an attractive tail, brings forth two distinct fluid phases into the system. Likewise, to observe a pressure-driven solid-solid transition at $T=0, v(r)$ must have a shoulder before the minimum [8, 11, 12]; with this trick, the minimum-energy configuration (which is dominant at low temperatures and pressures) is kept distinct from the close-packed configuration (which is preferred at high pressures). Yet, these two requirements alone do not imply a unique potential, since the extent of the hard core (i.e., the particle diameter) as well as the height and width of the shoulder relative to the well remain at will. The following potentials, defining the LG34 and LG56 models, are just two of a host of choices:

$$
v_{34}(r)=\left\{\begin{aligned}
+\infty, & \text { for } r \leq r_{2} \\
0, & \text { for } r=r_{3} \\
-\epsilon, & \text { for } r=r_{4} \\
0, & \text { for } r \geq r_{5}
\end{aligned} \quad \text { and } \quad v_{56}(r)=\left\{\begin{aligned}
+\infty, & \text { for } r \leq r_{4} \\
0, & \text { for } r=r_{5} \\
-\epsilon, & \text { for } r=r_{6} \\
0, & \text { for } r \geq r_{7},
\end{aligned}\right.\right.
$$

where $r_{n}$ is the distance between a pair of $n$-th neighbor lattice sites and $\epsilon>0$ sets the temperature scale. A pictorial description of these models can be found in Fig. 1.

To determine whether an IM is possible or definitely excluded in a lattice gas with two distinct solid ground states, the low-temperature profile of the solid-solid $P_{\operatorname{cox}}(T)$ is constructed, looking for a downward bending near $T=0$. However, a negative slope is not sufficient evidence of a genuine IM unless a continuous path exists from solid B to liquid, a question that can only be settled numerically, by e.g. the transfer-matrix method. 
I recall that, for given temperature $T$ and chemical potential $\mu$, the equilibrium state of a system with fixed volume $V$ is the one minimizing the generalized thermodynamic potential $\tilde{\Omega}=E-T S(E, V, N)-\mu N$ as a function of energy $E$ and particle number $N, S(E, V, N)$ being the system entropy function [13]. The minimum $\tilde{\Omega}$ defines the system grand potential $\Omega$, whereas $-\Omega / V$ is the equilibrium pressure $P$ expressed in terms of $T$ and $\mu$. At $T=0$, the eligible configurations of a lattice gas are only a few, each continuously linked with a possible system phase at $T>0 . T=0$ transitions occur in coincidence with any jump in the values of $E$ and $N$ at the point of minimum of $\tilde{\Omega}$.

In the LG56 model, for example, only three states are involved at $T=0$, namely two triangular crystals (solid A and solid B) and the vacuum (the $T=0$ vapor) - other solid phases, with rectangular rather than triangular symmetry, are excluded. With a slight abuse of terminology, the same three phases are hereafter termed solid, liquid, and vapor (in other words, I use "solid B" and "liquid" interchangeably as synonyms of a low-temperature loosepacked phase). In the liquid phase, the number density $\rho$ takes values that are intermediate between those of solid and vapor. To set the notation, let $a$ be the triangular-lattice spacing and $v_{\mathrm{c}}=(\sqrt{3} / 4) a^{2}$ the elementary-cell volume - the total number of lattice sites is $M=$ $V / v_{\mathrm{c}}$. The $T=0$ values of $\Omega / M$ for the three phases are $-\mu / 9,(-3 \epsilon-\mu) / 12$, and 0 , respectively. Whence a liquid-vapor transition at $\mu=\mu_{\mathrm{LV}} \equiv-3 \epsilon$, followed by a solid-liquid transition at $\mu=\mu_{\mathrm{SL}} \equiv 9 \epsilon$. The coexistence pressures in reduced, $\epsilon / v_{\mathrm{c}}$ units are $P_{\mathrm{LV}}^{*}=0$ and $P_{\mathrm{SL}}^{*}=1$, respectively. A similar treatment for the LG34 model yields $T=0$ phase transitions at $\mu=-3 \epsilon$ and $\mu=4 \epsilon$, with the same reduced values of the coexistence pressures as for LG56.

In order to extend the analysis to non-zero (small) temperatures, I proceed as follows. For each phase of the model, I list the allowed configurations (i.e., with no overlapping particles) that are obtained from the ground state by removing or adding few particles. Afterwards, for each excited state I calculate the multiplicity and the Boltzmann weight in terms of $M, \beta \mu$, and $\beta \epsilon\left(\beta=\left(k_{B} T\right)^{-1}\right.$, where $k_{B}$ is Boltzmann's constant). Finally, for each pair of coexisting phases, particle configurations are ordered by increasing relevance close to the transition threshold. The goal is to come up with an approximate expression for the $\Omega$ of each phase that could next be used to draw the low-temperature portion of the transition lines.

The detailed working out of these truncated expansions is rather lengthy. In the Ap- 
pendix, only a sketch of the derivation is presented, using the LG56 model as an example. Besides that, also the relevant expansions for the LG34 model are reported. For both models, the solid-liquid $P_{\text {cox }}(T)$ shows a decreasing behavior at low temperature, leaving room for an IM in both cases (see Figs. 9-12). While postponing to Section 4 the integration of these results with the full transfer-matrix data, I here comment on the mechanism that is responsible for a larger entropy of the solid phase at coexistence. As specifically illustrated in the Appendix for the LG56 model, in the liquid phase there is a strong imbalance between vacancies and interstitials as for free-energy cost: At low temperature, vacancies are far more easily excited than interstitials, which instead require a considerable rearrangement of the local structure in order to make room for the extra particles. On the other hand, vacancies have a lower cost in the solid phase since any particle removal in the liquid goes along with the rupture of many nearest-neighbor bonds, hence with a substantial energy increase. The outcome is that, at $T>0$, a smaller pressure than at zero temperature is required for stabilizing the solid phase.

\section{METHOD}

To reconstruct the phase diagram of a lattice-gas model, I use the transfer-matrix method and, to some extent, also grand-canonical Monte Carlo (MC). If the interaction has an upper cutoff, the exact grand potential of a system being infinite in one spatial direction and finite in the other(s) can be computed as the logarithm of the maximum eigenvalue $\lambda_{\max }$ of a so called transfer matrix (TM). In two dimensions, the simplest case occurs when this matrix encodes the interaction between a row of sites and the next row along the infinite strip direction $y$. In this case, the matrix size equals the total number of states in a row. More generally, depending on the interaction range, the natural lattice unit (NLU) can consist of

just one single row, or a pair of consecutive rows, or a triplet of rows, etc. In terms of $\lambda_{\max }$, the pressure of the strip reads:

$$
P=\frac{k_{B} T}{\mathcal{N}_{\mathrm{NLU}}} \ln \lambda_{\max },
$$

$\mathcal{N}_{\text {NLU }}$ being the number of sites in the NLU. The number density $\rho=(\partial P / \partial \mu)_{T}$ and its $\beta \mu$ derivative, related to the isothermal compressibility by $K_{T}=\rho^{-2}(\partial \rho / \partial \mu)_{T}$, are evaluated from the raw $\beta P$ data as a three-point numerical first- and second-order derivative, respectively ( $\beta \mu$ is made to increase by steps of 0.005$)$. As a rule, the number of iterations 
that are necessary to bring the maximum TM eigenvalue to convergence by the power method is larger the larger $K_{T}$. Upon increasing the number $N_{x}$ of sites in a row, phase-transition signatures - in the form of peaks in $(\partial \rho / \partial \mu)_{T}$ - gradually emerge, allowing to extract the infinite-size behavior. The virtue of the TM method is only limited by the range of the potential and by its core extension, which both determine the maximum $x$ size that can be stored in the computer.

The TM study of two-dimensional (2D) lattice gases received a strong impulse in the mid sixties by the work of Runnels and coworkers [14, 15]: These authors provided a considerable simplification in the problem by showing how to reduce the TM size substantially without affecting $\lambda_{\max }$. In practice, NLU states are grouped into equivalence classes bringing together states that map onto each other when translated along $x$ and/or reflected with respect to the strip axis. Whence, a matrix that is a condensed form of the TM is defined, of size equal to the number of equivalence classes, whose maximum eigenvalue is the same as for the original TM. In Table 1, some typical dimensions are reported for the original TM and for its compactified form, with reference to the LG34 and LG56 models. Among many similar models, LG34 and LG56 provided the right compromise between large core extension and feasibility of the TM analysis for quite large sizes.

I have complemented the TM study with Metropolis MC simulations in the grandcanonical ensemble. Typically, two million MC sweeps are generated at equilibrium for $L \times L$ triangular lattices of increasing size, up to a maximum of $L=240$, with periodic boundary conditions. A MC sweep consists of one average attempt per site to change the occupation number. As a rule, simulation runs are carried out in a sequence, starting at a low $\beta \mu$ value from the empty lattice and then raising $\beta \mu$ progressively at fixed temperature. In the region of solid-liquid coexistence, equilibrium sampling could be obstructed by liquid undercooling, i.e., by hysteresis. This is why, at high densities, the TM method (where available) is to be preferred to MC simulations.

Among the computed quantities, the reduced number density $\rho^{*} \equiv \rho v_{\mathrm{c}}$ and the isothermal compressibility $K_{T}$ are especially monitored. These are expressed in terms of grand-canonical averages as

$$
\rho^{*}=\langle\mathcal{N}\rangle / L^{2} \quad \text { and } \quad \rho k_{B} T K_{T}=\frac{\left\langle(\Delta \mathcal{N})^{2}\right\rangle}{\langle\mathcal{N}\rangle},
$$

with $\Delta \mathcal{N}=\mathcal{N}-\langle\mathcal{N}\rangle, \mathcal{N}$ being the current particle number. 
Finally, a useful tool for identifying the order of a phase transition is to follow, e.g. for given $\beta$, the evolution of the $\mathrm{MC}$ density histogram as a function of $\mu$. In a finite system, a roughly Gaussian peak in this histogram is the imprint of a homogeneous phase, a secondorder transition (or just a crossover) is signalled by a broader peak, while phase coexistence appears as a bimodal density distribution. Hence, it is possible to discriminate between smooth/continuous and first-order condensation by just looking at the $\mu$-evolution of the density histogram at fixed temperature.

\section{RESULTS}

\section{A. LG34 model}

This model was studied with the TM method only, considering just one lattice strip 14 sites wide. This is the smallest size allowing both solid ground states to be accommodated into the strip. The next suited size, 28, is just too big for being amenable to numerical analysis by the TM method.

In Fig. 2, I show results for three isotherms somehow representative of the different regimes, the overall phase diagram being represented in Fig. 3. It is clear that, beyond a fluid phase and a solid of (reduced) density $\approx 1 / 4$, there is also an intermediate solid $\mathrm{B}$ phase, of density $\approx 1 / 7$, which is present only at low temperature. As temperature grows, the border which marks the region of solid B stability gets thinner till it disappears, leaving a passage into the fluid. However, this is an effect of the finite strip size while, in the thermodynamic limit, there will likely be no way to enter solid B smoothly from the fluid. Hence, calling "solid" the phase with $\rho^{*} \approx 1 / 7$ (rather than "liquid") is a proper usage. Finally, the fluid phase shows vapor-like properties at low pressure, with densities lower than $1 / 7$, while appearing liquid-like for high pressures, with densities higher than $1 / 7$.

The two solids and the liquid meet in a triple point at $T^{*} \equiv k_{B} T / \epsilon \simeq 0.90$ and $P^{*} \equiv$ $P v_{\mathrm{c}} / \epsilon \simeq 0.98$. Above this pressure, and up to $P^{*}=1$, the solid-solid transition under isobaric conditions anomalously occurs with volume contraction on heating, as already anticipated from the low-temperature expansions.

Though the reported data are for just one strip size, their consistency with an exact lowtemperature analysis (see Figs. 11 and 12) and the sharpness of the transition signatures 
make me confident that the features observed in Fig. 3 give a faithful account of the phase behavior of the model in the thermodynamic limit.

The LG34 model resembles the phase behavior of the purely repulsive lattice-gas model studied in [16], hereby called LG3, where particles exclude first- and second-neighbor sites on a triangular lattice while softly repelling each other at third-neighbor distance. Both models share the same phases, although LG34 is more effective in promoting the stability of the solids at the expense of the fluid. Moreover, the possibility of a solid-solid transition at constant pressure, further accompanied by a density increase on heating, is only peculiar to the LG34 model.

\section{B. LG56 model}

With respect to LG34, hard-core exclusion now comprises also third- and fourth-neighbor sites. $N_{x}$ must be a multiple of 6 in order that both solid ground states fit into the periodized strip. Fig. 4 shows a selection of results, relative to a number of isothermal paths for $N_{x}=18$. At low temperature, there is a clear two-stage transition from vapor to solid A, with an intermediate phase of density $\approx 1 / 12$. This phase has strong crystalline features, as signalled by the extremely small values of $K_{T}$, hence it will tentatively be called solid B.

Upon increasing the temperature, the character of the first transition gradually modifies, becoming smoother and smoother until it disappears for $\beta \epsilon \approx 0.38$, leaving a direct transition from vapor to solid A at higher temperatures. At the same time, the nature of the intermediate phase also changes, since appreciable $\left(0.1\right.$ or so) values of $\partial \rho^{*} / \partial \beta \mu$ give support to the idea that this phase is actually liquid (I shall add more evidence on this later). The resulting phase diagram, see Fig. 5, resembles that of ${ }^{4} \mathrm{He}$, with the obvious difference that there is no superfluid region in the LG56 liquid. The most exciting feature of the LG56 phase diagram is the clear IM behavior that is found in the narrow pressure range from $\approx 0.97$ to 1 (see Fig. 5 inset). The mechanism upon which IM rests has been already

clarified in Section 2: it deals with the lower cost of proliferating vacancies in solid A than in the low-temperature solid B.

In the same Fig. 5, I sketch the phase diagram of another model, called LG5. Its pair potential shares the same core with $v_{56}(r)$, but it shows a repulsive $\epsilon$ shoulder at $r=r_{5}$ and no well. This purely-repulsive model cannot have a liquid phase, hence the $\rho^{*} \approx 1 / 12$ phase 
should be a triangular solid which, in the thermodynamic limit, will be separated from the fluid phase by an uninterrupted first-order line. The IM-like feature that is seen along the solid-solid coexistence line of LG5 is probably an artefact of the finite strip size, since it deflates upon going from $N_{x}=12$ to 18 .

To probe the exactness of the TM technique, I have carried out a series of MC simulations for a $18 \times 360$ lattice at $\beta \epsilon=0.4$. MC data points, reported as dots and asterisks in Fig. 4, do clearly lie superimposed over the TM data. When the temperature is sufficiently high, a more significant check of the TM results is provided by the calculation of the system pressure with the method of thermodynamic integration. At high temperatures, the simulation can be pushed through the transition region between liquid and solid, with no risk of bumping into phase-space bottlenecks (in other words, no hysteresis or effective ergodicity breaking is observed). Eventually, $\beta \mu$ becomes so high that the truncated expansion (A.10) of the LG56 solid pressure holds true. Combining this expansion with the MC values of the number density, I was able to obtain an estimate of the pressure at the point of maximum of $\partial \rho^{*} / \partial \beta \mu$ (the asterisk in Fig. 5 inset) that compares well with the TM datum.

The main question left open by the TM analysis concerns the nature of the $\rho^{*} \approx 1 / 12$ phase: Is it actually a liquid? A related question is: Does the liquid-vapor boundary of the infinite-size system really terminate near $\beta \epsilon=0.38$ or it will rather join somewhere to the freezing line, like in the LG5 case? Should the latter be true, it would indeed be hard to qualify the intermediate phase of the LG56 model as liquid.

I have carried out MC simulations of large $L \times L$ triangular lattices, up to $L=240$, along three isotherms, $\beta=030,0.34$, and 0.38 . Each series of runs was arrested at the edge of freezing, i.e., before equilibrium statistics could become unefficiently sampled by MC. The values obtained for the number density and its $\beta \mu$ derivative are reported in Fig. 6, together with the TM data for $N_{x}=18$. While leaving substantially unaltered (with respect to the TM hint) the location of freezing, the MC results clearly indicate that vapor condensation persists well beyond $T^{*}=1 / 0.38$, without merging into the freezing transition (see the asterisks in Fig. 7).

Further information on vapor condensation can be acquired by monitoring the density histogram as a function of $\beta \mu$ at constant temperature. This is drawn in Fig. 7 inset for $L=240$ and $\beta \epsilon=0.38$ (similar results are found for $\beta \epsilon=0.34$ and 0.30). Note the same Gaussian character and the comparable width of the density histograms on either side 
of the condensation point. While a first-order condensation can be safely excluded from these results, it would be hard to discriminate a locus of critical points from a disorder line. The numerical errors affecting particle-number fluctuations are not small enough to decide, from the size scaling of the compressibility maximum, whether the condensation line terminates with a critical ending point or rather proceeds to infinite temperature. In any case, a smooth vapor condensation can hardly be reconciled with the symmetry breaking that would be implied by a phase transition into a triangular solid. It is true that there are lattice gases where the transition from vapor to solid is reported to be second-order or even smoother, but this only occurs when the core diameter of the particles is very small [17]. Moreover, the large values of $\partial \rho^{*} / \partial \beta \mu$ within the $\beta \mu$ range of the intermediate phase are more appropriate to a liquid than to a solid.

There is a last point to discuss, related to the possibility that a phase-transition line separating solid B from liquid, running at about constant $T$, was overlooked by the present TM study, which considered only isothermal scans of the phase diagram. It is worth noting that this was not the case for the LG34 model, where in fact the TM analysis revealed the existence of a clear first-order boundary between the two phases. The question remains as to whether a smoother transition occurs in the LG56 case. To clarify this point, I carried out a TM study of the $N_{x}=12$ and 18 strips along various constant- $\mu$ lines. The locus of points where $\partial \rho^{*} / \partial \beta \mu$ is maximum as a function of temperature is reported as a dashed line in Fig. 7. By looking at this picture, one is tempted to conclude that solid B and liquid are actually distinct phases. In fact, things are more complicate since, for e.g. $\mu=8 \epsilon$, the broad maximum of $\partial \rho^{*} / \partial \beta \mu$ occurs with no evidence of density jump - see Fig. 8. My conclusion is that either this maximum marks the crossover from a prominently liquid-like to a prominently solid B-like behavior within the same phase or there is an underlying weak first-order transition between liquid and solid B, characterized by a small jump of specific volume. In the latter case, the seemingly negative slope of the phase boundary would be the result, via Eq. (2.1), of a slight number-density decrease occurring upon going from liquid to solid B, which is consistent with the TM data of Fig. 8.

Further information is obtained from a series of MC simulations that I carried out for $\mu=8 \epsilon$, with $\beta \epsilon$ ranging from 0.3 to 0.4 . In Fig. 8, I report results for two sizes, $L=120$ and 240. It turns out that the only clear singularity occurs at $\beta \epsilon \simeq 0.34$, which corresponds to the same liquid-vapor transition found at $\beta \mu \simeq 2.70$ along the $\beta \epsilon=0.34$ isotherm. 
However, upon increasing $\beta \epsilon$ a little further, the simulated system abruptly transformed into an almost perfect realization of solid B, suggesting that what probably realizes in the LG56 model is the weak-transition scenario: The low-temperature liquid, which looks like a disordered patchwork of solid A and solid B grains, is not capable to dismiss its solid A fraction continuously on cooling, being thus forced to transform into solid B abruptly. Considering that the hypothetical solid B-liquid boundary appears to join to the locus of solid A melting at about where the slope of the latter changes from negative to positive, the IM behavior of the LG56 model might not differ significantly from the LG34 model, though the former is undoubtedly closer to realize the ideal IM scenario than the latter.

Finally, let me draw some implications from the above results. From the arguments presented in the Appendix, it is evident that a decreasing freezing line on the $T$ - $P$ plane will be the rule, at sufficiently low temperature, for all the potentials having the same shape of $v_{56}(r)$. Whether this is an imprint of a genuine IM, rather than of a solid-solid transition occurring with volume contraction upon heating at constant pressure, is a complicate matter to grasp, which might be linked to the existence of a congruous number of liquid configurations that are proximal, as for number density, to the loose-packed crystalline ground state. This is about to occur in the LG56 case, where the equilibrium liquid at moderately low temperatures comes indeed very close, as for specific volume and entropy contents, to a defected solid B. Supposedly, when both particle core and potential well become slightly enlarged with respect to the LG56 case, with the width of the barrier staying fixed, the likelihood of observing a genuine IM will get enhanced.

\section{CONCLUSIONS}

Inverse melting (IM) is the phenomenon by which a crystal melts when cooled at constant pressure. This can only occur if, at the transition point, the solid is more entropic than the liquid. Besides Helium at low temperature, the only system where an IM-like transition is observed are some peculiar polymer solutions, denoted P4MP1.

In the present paper, I investigated the possibility of a lattice analog of IM. To this aim, I introduced a triangular-lattice-gas system, called LG56, where the particle diameter is 3, in units of the lattice constant, and there is a narrow attractive well at a bit larger distance, $2 \sqrt{3}$ lattice units. The phase diagram of this system bears some indication of IM, similar 
to that occurring in Helium by a completely different mechanism, which can be rationalized as follows: The soft inter-particle repulsion causes the existence of two distinct crystalline ground states, a close-packed solid at high pressures and a more open crystal structure at low pressures. When the core diameter is large enough, which is about the case of the LG56 model, the loose-packed crystal will smoothly transform into a liquid at high temperatures, i.e., without crossing a neat phase boundary. In addition to that, the interplay between interaction and lattice geometry produces two effects, both essential to promote IM: 1) Interstitials are heavily suppressed in the low-temperature liquid; 2) vacancies are more easily excited in the solid than in the liquid phase, thus conferring an entropic benefit to the solid. As a result, the melting line on the $T$ - $P$ plane bends downward at sufficiently low temperature.

It can be argued that a genuine IM cannot be observed in a softly-repulsive lattice-gas system since, upon lowering the temperature, any dense fluid phase should eventually turn into a loose-packed solid. In fact, the real question is whether a stable liquid can be pushed to such low temperatures that, in a range of pressures, the close-packed solid first melts into the liquid upon cooling, only after transforming into the loose-packed solid. The present study shows that the LG56 model is indeed close to realize this ideal IM scenario.

I add a final remark on the transferability of the above results to soft-core potentials on a 3D lattice. As far as the previously cited requisites on the inner-core and attractive-well extensions are met, I find no reason to think that the behavior in $3 \mathrm{D}$ will be much different. In particular, the same mechanism leading in 2D to a minimum in the freezing pressure as a function of temperature will be at work also in 3D. Presumably, the only marked difference concerns the order of the phase transitions, which are stronger in a higher-dimensionality space. Consider, for instance, a 3D counterpart of the LG56 model. While the freezing, solid A-to-liquid transition of the LG56 model is strongly first-order already in 2D, the transition from solid B to liquid would be much neater in 3D, with the effect of removing any residual ambiguity on the nature of the dashed-line crossover of Fig. 7.

\section{Acknowledgements}

This work was supported by the University of Messina. I wish to express my thanks to Paolo V. Giaquinta for introducing me to the fascinating field of inverse melting and for a critical reading of the manuscript. I am also grateful to Dora Magaudda at CECUM for allowing 
me to use the "Eneadi" computer cluster prior to its full implementation. 


\section{APPENDIX A: LOW-TEMPERATURE EXPANSIONS}

In this Appendix, exact series expansions are used to examine the low-temperature behavior of the LG34 and LG56 models, with specific regard to the determination of first-order phase boundaries. Besides their intrinsic interest, these expansions may provide a consistency check of the TM results. For both lattice gases, an expansion of the grand partition function $\Xi$, from which the grand potential $\Omega$ follows as $-k_{B} T \ln \Xi$, is presented for all low-temperature phases: solid, liquid, and vapor (in this appendix, I call "liquid" what is actually the loose-packed solid B phase). For a pair of competing phases, the transition line is located where the respective grand potentials are equal. The derivation of these expansions is rather lengthy and it would demand too space to be reported here with full detail, hence it will be just sketched. For one model (LG56), the high- $\mu$ expansion of the solid pressure is also displayed.

LG56 model. - In the very cold vapor, there are just a few particles on an otherwise empty lattice. Leading terms in $\Xi$ are those associated with the largest Boltzmann factors, hence with a small number of particles, better if linked by liquid-like bonds. The expansion of $\Xi_{\mathrm{V}}$ will appear as:

$$
\begin{aligned}
\Xi_{\mathrm{V}} & =1+M e^{\beta \mu}+\frac{M(M-37)}{2} e^{2 \beta \mu}+3 M e^{2 \beta \mu} e^{\beta \epsilon}+3 M(M-61) e^{3 \beta \mu} e^{\beta \epsilon}+9 M e^{3 \beta \mu} e^{2 \beta \epsilon} \\
& +2 M e^{3 \beta \mu} e^{3 \beta \epsilon}+12 M e^{4 \beta \mu} e^{4 \beta \epsilon}+3 M e^{4 \beta \mu} e^{5 \beta \epsilon}+\ldots
\end{aligned}
$$

The prefactors of the exponentials are multiplicities as calculated for the bulk system.

Similarly, in the very cold liquid, the microstates which occur with higher probability at equilibrium are obtained from the triangular crystal of density 1/12 by removing a small number of particles, better if bound to each other. The grand partition function starts with:

$$
\begin{aligned}
\Xi_{\mathrm{L}} & =e^{(\beta \mu+3 \beta \epsilon) M / 12}\left[12+M e^{-\beta \mu} e^{-6 \beta \epsilon}+3 M e^{-\beta \mu} e^{-11 \beta \epsilon}+3 M e^{-2 \beta \mu} e^{-11 \beta \epsilon}\right. \\
& +\frac{M}{2}\left(\frac{M}{12}-7\right) e^{-2 \beta \mu} e^{-12 \beta \epsilon}+2 M e^{-3 \beta \mu} e^{-15 \beta \epsilon}+9 M e^{-3 \beta \mu} e^{-16 \beta \epsilon} \\
& +3 M\left(\frac{M}{12}-10\right) e^{-3 \beta \mu} e^{-17 \beta \epsilon}+3 M e^{-4 \beta \mu} e^{-19 \beta \epsilon}+12 M e^{-4 \beta \mu} e^{-20 \beta \epsilon} \\
& \left.+6 M e^{-5 \beta \mu} e^{-23 \beta \epsilon}+\ldots\right] .
\end{aligned}
$$

Close to $\mu_{\mathrm{LV}}=-3 \epsilon$ and $T=0$, I set $\beta \mu=-3 \beta \epsilon+\delta$. Moreover, I define the small 
parameter $x=\exp (-\beta \epsilon)$. Disregarding all terms smaller than $x^{8}$, one eventually finds:

$$
\frac{\ln \Xi_{\mathrm{V}}}{M}=e^{\delta} x^{3}+3 e^{2 \delta} x^{5}+\left(2 e^{3 \delta}-\frac{37}{2} e^{2 \delta}\right) x^{6}+\left(3 e^{4 \delta}+9 e^{3 \delta}\right) x^{7}+\left(12 e^{4 \delta}-183 e^{3 \delta}\right) x^{8}+\ldots
$$

and

$$
\begin{aligned}
\frac{\ln \Xi_{\mathrm{L}}}{M} & =\frac{\delta}{12}+\frac{1}{12} e^{-\delta} x^{3}+\frac{1}{4} e^{-2 \delta} x^{5}+\left(-\frac{7}{24} e^{-2 \delta}+\frac{1}{6} e^{-3 \delta}\right) x^{6}+\left(\frac{3}{4} e^{-3 \delta}+\frac{1}{4} e^{-4 \delta}\right) x^{7} \\
& +\left(\frac{1}{4} e^{-\delta}-\frac{5}{2} e^{-3 \delta}+e^{-4 \delta}+\frac{1}{2} e^{-5 \delta}\right) x^{8}+\ldots
\end{aligned}
$$

In these formulae, we see the linked-cluster theorem at work: Despite the fact that multiplicities of disconnected clusters of defects are not linear in $M$, see (A.1) and (A.2), the logarithm of the partition function turns out to be extensive due to cancellation of terms proportional to $M^{2}, M^{3}$, etc.

For given $\beta$ and $\beta \mu$, the stable phase has the largest $\Xi$ or, equivalently, $\ln \Xi$. Stated differently, the vapor is more stable than the liquid as far as $\Omega_{\mathrm{V}}<\Omega_{\mathrm{L}}$, or

$$
\begin{aligned}
\delta e^{\delta} & <\left(12 e^{2 \delta}-1\right) x^{3}+\left(36 e^{3 \delta}-3 e^{-\delta}\right) x^{5}+\left(24 e^{4 \delta}-222 e^{3 \delta}+\frac{7}{2} e^{-\delta}-2 e^{-2 \delta}\right) x^{6} \\
& +\left(36 e^{5 \delta}+108 e^{4 \delta}-9 e^{-2 \delta}-3 e^{-3 \delta}\right) x^{7} \\
& +\left(144 e^{5 \delta}-2196 e^{4 \delta}-3+30 e^{-2 \delta}-12 e^{-3 \delta}-6 e^{-4 \delta}\right) x^{8}+\ldots
\end{aligned}
$$

In particular, for small $x$ and $\delta=0$ the stable phase is vapor, meaning that the low- $T$ part of the liquid-vapor coexistence line is bent upward in the $\mu$ - $T$ plane, see a comparison with the TM data in Fig. 9 below.

As far as the solid is concerned, the low-cost excitations are defected crystals with a small number of vacancies. The expansion of the grand partition function starts with:

$$
\Xi_{\mathrm{S}}=e^{\beta \mu M / 9}\left[9+M e^{-\beta \mu}+\frac{M}{2}\left(\frac{M}{9}-1\right) e^{-2 \beta \mu}+2 M e^{-2 \beta \mu} e^{3 \beta \epsilon}+3 M e^{-3 \beta \mu} e^{7 \beta \epsilon}+\ldots\right] .
$$

For the liquid, I can still use Eq. (A.2) since interstitials are irrelevant at low temperature: Their accomodation requires such a huge reorganization of the local liquid structure, along with the breaking of so many bonds, that the loss in Boltzmann weight due to a higher energy greatly overcomes the gain due to an increased particle number. Close to $\mu_{\mathrm{LS}}=9 \epsilon$ and $T=0$, I set $\beta \mu=9 \beta \epsilon+\delta$ and $x=\exp (-\beta \epsilon)$, thus arriving at the following $\ln \Xi$ expansions for the liquid and the solid:

$$
\frac{\ln \Xi_{\mathrm{L}}}{M}=\beta \epsilon+\frac{\delta}{12}+\frac{1}{12} e^{-\delta} x^{15}+\frac{1}{4} e^{-\delta} x^{20}+\ldots
$$


and

$$
\frac{\ln \Xi_{\mathrm{S}}}{M}=\beta \epsilon+\frac{\delta}{9}+\frac{1}{9} e^{-\delta} x^{9}+\frac{2}{9} e^{-2 \delta} x^{15}-\frac{1}{18} e^{-2 \delta} x^{18}+\frac{1}{3} e^{-3 \delta} x^{20}+\ldots
$$

The solid is more stable than the liquid when $\Omega_{\mathrm{S}}<\Omega_{\mathrm{L}}$, or

$$
\delta e^{\delta}>-4 x^{9}+\left(3-8 e^{-\delta}\right) x^{15}+2 e^{-\delta} x^{18}+\left(9-12 e^{-2 \delta}\right) x^{20}+\ldots
$$

For small $x$, the above inequality is satisfied for $\delta=0$, indicating that the solid-liquid coexistence line is bent downward at small temperature (see Fig. 9 above). This holds true also on the $T$ - $P$ plane, as one obtains from the values of the liquid or solid $P^{*}=T^{*} \ln \Xi / M$ along the coexistence line $\mu_{\text {cox }}(T)$, see Fig. 10 above.

Finally, the first few terms in the high- $\mu$ expansion of the solid pressure for the LG56 model are:

$$
\beta P v_{c}=\frac{\beta \mu}{9}+\frac{1}{9} e^{-\beta \mu}+\frac{4 e^{3 \beta \epsilon}-1}{18} e^{-2 \beta \mu}+\left(\frac{1}{3} e^{7 \beta \epsilon}-\frac{2}{3} e^{3 \beta \epsilon}+\frac{19}{27}\right) e^{-3 \beta \mu}+\ldots
$$

From here, the high- $\mu$ expansion of the number density follows by differentiation.

LG34 model. - Without entering much into details, which are similar as for LG56, I list the expansions which are relevant for the low-temperature analysis of the LG34 model.

The grand partition function of the vapor reads:

$$
\begin{aligned}
\Xi_{\mathrm{V}} & =1+M e^{\beta \mu}+\frac{M(M-25)}{2} e^{2 \beta \mu}+6 M e^{2 \beta \mu} e^{\beta \epsilon}+6 M(M-40) e^{3 \beta \mu} e^{\beta \epsilon}+42 M e^{3 \beta \mu} e^{2 \beta \epsilon} \\
& +4 M e^{3 \beta \mu} e^{3 \beta \epsilon}+60 M e^{4 \beta \mu} e^{4 \beta \epsilon}+6 M e^{4 \beta \mu} e^{5 \beta \epsilon}+\ldots
\end{aligned}
$$

The grand partition function of the liquid (not to be confused with the dense fluid phase of the model) reads:

$$
\begin{aligned}
\Xi_{\mathrm{L}} & =e^{(\beta \mu+3 \beta \epsilon) M / 7}\left[14+2 M e^{-\beta \mu} e^{-6 \beta \epsilon}+12 M e^{-\beta \mu} e^{-10 \beta \epsilon}+6 M e^{-2 \beta \mu} e^{-11 \beta \epsilon}\right. \\
& +M\left(\frac{M}{7}-7\right) e^{-2 \beta \mu} e^{-12 \beta \epsilon}+12 M e^{-2 \beta \mu} e^{-14 \beta \epsilon}+4 M e^{-3 \beta \mu} e^{-15 \beta \epsilon}+18 M e^{-3 \beta \mu} e^{-16 \beta \epsilon} \\
& +6 M\left(\frac{M}{7}-10\right) e^{-3 \beta \mu} e^{-17 \beta \epsilon}+6 M e^{-4 \beta \mu} e^{-19 \beta \epsilon}+24 M e^{-4 \beta \mu} e^{-20 \beta \epsilon} \\
& \left.+12 M e^{-5 \beta \mu} e^{-23 \beta \epsilon}+\ldots\right] .
\end{aligned}
$$

With the same $x$ as before, the insertion of $\beta \mu=-3 \beta \epsilon+\delta$ into Eqs. (A.11) and (A.12) 
shows that the vapor is more stable than the liquid when

$$
\begin{aligned}
\delta e^{\delta} & <\left(7 e^{2 \delta}-1\right) x^{3}+\left(42 e^{3 \delta}-3 e^{-\delta}\right) x^{5}+\left(28 e^{4 \delta}-\frac{175}{2} e^{3 \delta}+\frac{7}{2} e^{-\delta}-2 e^{-2 \delta}\right) x^{6} \\
& +\left(42 e^{5 \delta}+294 e^{4 \delta}-6-9 e^{-2 \delta}-3 e^{-3 \delta}\right) x^{7} \\
& +\left(420 e^{5 \delta}-1680 e^{4 \delta}-6 e^{-\delta}+30 e^{-2 \delta}-12 e^{-3 \delta}-6 e^{-4 \delta}\right) x^{8}+\ldots
\end{aligned}
$$

The ensuing liquid-vapor coexistence line on the $T$ - $\mu$ plane is reported in Fig. 11 below.

The solid grand partition function reads:

$$
\Xi_{\mathrm{S}}=e^{\beta \mu M / 4}\left[4+M e^{-\beta \mu}+\frac{M}{2}\left(\frac{M}{4}-1\right) e^{-2 \beta \mu}+3 M e^{-3 \beta \mu} e^{4 \beta \epsilon}+\ldots\right] .
$$

Upon substituting $\beta \mu=4 \beta \epsilon+\delta$ into Eqs. (A.12) and (A.14), it finally turns out that the solid is more stable than the liquid when

$$
\delta e^{\delta}>-\frac{7}{3} x^{4}+\left(\frac{7}{6} e^{-\delta}-7 e^{-2 \delta}\right) x^{8}+\frac{4}{3} x^{10}+\ldots
$$

The solid-liquid coexistence line is reported in Fig. 11 above. Phase boundaries on the T-P plane have a similar appearance, see Fig. 12.

[1] See, for instance, S. Balibar, H. Alles, and A. Y. Parshin, Rev. Mod. Phys. 77, 317 (2005).

[2] S. Rastogi, G. W. H. Hohne, and A. Keller, Macromolecus 32, 8897 (1999); A. L. Greer, Nature 404, 134 (2000).

[3] G. Tammann, Kristallisieren und Schmelzen, Verlag Johann Ambrosius Barth, Leipzig, 1903.

[4] F. H. Stillinger, P. G. Debenedetti, and T. M. Truskett, J. Phys. Chem. B 105, 11809 (2001); F. H. Stillinger and P. G. Debenedetti, Biophys. Chem. 105, 211 (2003).

[5] M. R. Feeney, P. G. Debenedetti, and F. H. Stillinger, J. Chem. Phys. 119, 4582 (2003).

[6] N. Schupper and N. M. Shnerb, Phys. Rev. Lett. 93, 037202 (2004); Phys. Rev. E 72, 046107 (2005).

[7] For a review, see L. K. Runnels, in Phase Transitions and Critical Phenomena, edited by C. Domb and M. S. Green (Academic, London, 1972), Vol. 2.

[8] J. Orban, J. Van Craen, and A. Bellemans, J. Chem. Phys. 49, 1778 (1968).

[9] D. Poland, Phys. Rev. E 59, 1523 (1999).

[10] S. Prestipino, Phys. Rev. E 62, 2177 (2000). 
[11] N. B. Wilding and J. E. Magee, Phys. Rev. E 66, 031509 (2002).

[12] D. Quigley and M. I. J. Probert, Phys. Rev. E 72, 061202 (2005).

[13] S. Prestipino and P. V. Giaquinta, J. Stat. Phys. 111, 479 (2003).

[14] L. K. Runnels and L. L. Combs, J. Chem. Phys. 45, 2482 (1966).

[15] L. K. Runnels, L. L. Combs, and J. P. Salvant, J. Chem. Phys. 47, 4015 (1967).

[16] L. K. Runnels, J. R. Craig, and H. R. Streiffer, J. Chem. Phys. 54, 2004 (1971).

[17] J. Orban and A. Bellemans, J. Chem. Phys. 49, 363 (1968). 


\section{TABLE CAPTION}

Table 1 : Some data concerning the TM of the lattice-gas models that are studied in this paper: $N_{x}$ is the number of lattice sites along the finite strip size, chosen in such a way as to comply with the periodicity of the two solid ground states (i.e., a multiple of 14 for LG34 and of 6 for LG56); $\mathcal{N}_{\mathrm{NLU}}$ gives the number of sites comprised in the natural lattice unit (i.e., $3 N_{x}$ for LG34 and $4 N_{x}$ for LG56); $M_{1}$ is the size of the original TM; $M_{2}$ is the size of the symmetry-reduced matrix, sharing the same leading eigenvalue with the original matrix. 


\section{FIGURE CAPTIONS}

Fig. 1 : Pictorial description of the lattice-gas models defined in Eq. (2.1). When a particle is placed in the position marked as a full dot, crosses denote forbidden sites for the centers of other particles. Open squares denote the lattice sites that are occupied by the nearest-neighbor particles of the central particle in the close-packed solid A. Open dots denote attractive sites for the central particle: Here are placed its nearestneighbor particles in the loose-packed solid B. In the LG34 model, these attractive sites are twelve, contributing a twofold degeneracy to the spatial orientation of solid B.

Fig. 2 : LG34 model, TM results for $N_{x}=14$. The reduced number density (dotted lines) and its $\beta \mu$ derivative (continuous lines) are shown for three isotherms, $\beta \epsilon=0.75,0.80$, and 0.89. To help the eye, straight lines are drawn between data points. Peaks in the density derivative are the imprint of phase boundaries: As a rule, the more pronounced a maximum is, the stronger the phase transition. The confluence of three distinct phases (solid $\mathrm{A}$, solid $\mathrm{B}$, and liquid) at $\beta \epsilon \approx 0.90$ is quite transparent from the behavior at $\beta \epsilon=0.89$ (see also Fig. 3 inset). Upon raising the temperature, the region of solid B gradually shrinks until its boundaries fade away. However, in the thermodynamic limit, there will reasonably be no path to go smoothly from fluid to solid B.

Fig. 3 : LG34 model, TM results for $N_{x}=14$. Overall phase diagram of the system as resulting from joining the $(T, P)$ points that correspond to $\partial \rho^{*} / \partial \beta \mu$ peaks. The LG34 potential (open dots and continuous lines) is contrasted with the LG3 repulsive law of Ref. [16] ( $N_{x}=14$, open squares and dashed lines). Spline interpolants drawn between LG34 data points mark first-order phase boundaries; the interruptions are finite-size effects. The fluid phase is vapor-like at low pressure and liquid-like at high pressure. While both models can exist in three phases (solid A, solid B, and fluid), LG34 is peculiar in that it shows a solid-solid transition with volume contraction on heating at constant pressure (inset).

Fig. 4 : LG56 model, TM results for $N_{x}=18$. The reduced number density (dotted lines) and its $\beta \mu$ derivative (continuous lines) are shown for a number of isotherms, $\beta \epsilon=$ 
$0.34,0.38,0.4,0.45,0.5,0.6$, and 0.7 . To help the eye, straight lines are drawn between data points. $\mathrm{MC}$ data for a $18 \times 360$ periodic sample, relative to $\beta \epsilon=0.4$, are also shown: Reduced number density (open dots) and its $\beta \mu$ derivative (asterisks), as computed through the number fluctuations - see Eq. (3.2). Upon increasing the temperature, the liquid-vapor peak progressively broadens till it disappears at $\beta \epsilon=$ 0.38. The solid-liquid peak is present at all temperatures: It is already very sharp for $\beta \epsilon=0.45$, becoming even sharper for lower temperatures (not shown).

Fig. 5 : LG56 model, TM phase diagram for $N_{x}=12$ (open triangles and dashed lines) and 18 (open dots and continuous lines). The phase diagram of LG56 is contrasted with that of LG5 ( $N_{x}=18$, open squares and dashed lines). Both models can exist in three phases: However, while the dense phase of low pressure is solid B for the purely-repulsive LG5 model, the analogous phase for LG56 is liquid, at least for high temperatures (see my arguments in Section 4.B). The LG56 model is peculiar in that an inverse melting occurs (inset). The asterisk is a point on the solid-liquid coexistence line of the wider LG56 strip that was constructed by MC (see main text). The shallow minimum in the solid-solid $P_{\operatorname{cox}}(T)$ of LG5 is probably a finite-size effect since this feature is less evident for $N_{x}=18$ than for $N_{x}=12$ (not shown).

Fig. 6 : LG56 model, data for three distinct isotherms, $\beta=0.30,0.34$, and 0.38. Above: reduced number density $\rho^{*}$; below: $\beta \mu$ derivative of $\rho^{*}$. TM data for $N_{x}=18$ (dotted lines) are contrasted with spline interpolants of the MC results for $L \times L$ lattices, with $L=120,180$, and 240 (continuous lines). The MC density derivative is computed through the number fluctuations via Eq. (3.2) (the larger $L$, the more pronounced the $\partial \rho^{*} / \partial \beta \mu$ maximum is). While distinctly recording the freezing transition at all temperatures, a strip of 18 sites is not large enough to follow the boundary between vapor and liquid beyond a reduced temperature of $1 / 0.38$. On the basis of the present $\mathrm{MC}$ evidence, it is hard to say whether the liquid-vapor boundary survives at all temperatures or it rather turns into a non-critical disorder line at a finite temperature (the scaling of the MC compressibility at the estimated transition point is inconclusive).

Fig. 7 : LG56 model, phase diagram on the $T$ - $\mu$ plane: TM results for $N_{x}=18$ (open dots and continuous lines) plus three MC data points for a $240 \times 240$ lattice (asterisks), corresponding to the location of the $\partial \rho^{*} / \partial \beta \mu$ maxima in Fig. 6. A further dashed line 
connects TM data points for $N_{x}=18$ recording maxima of $\partial \rho^{*} / \partial \beta \mu$ along constant- $\mu$ cuts. Inset: $\beta \mu$ evolution (from left to right) in the range from 2.30 to 3.25, with steps of 0.05 , of the density histogram for $\beta=0.38$ and $L=240$. It appears from the inset that the liquid-vapor transition either turns continuous at high temperatures or it becomes a crossover.

Fig. 8 : LG56 model, TM and MC data for the reduced number density $\rho^{*}$ and its $\beta \mu$ derivative along the $\mu=8 \epsilon$ line. TM data for $N_{x}=12$ (dashed line) and $N_{x}=18$ (dotted lines) are contrasted with MC results for $L \times L$ lattices, with $L=120$ and 240 (continuous lines). The transition at $\beta \epsilon \simeq 0.34$ is from vapor to liquid while a defected solid B structure abruptly appeared when pushing the simulation beyond $\beta \epsilon=0.40$. Inset: $\beta \mu$ evolution (from left to right) in the range from 0.3 to 0.4 , with steps of 0.005, of the density histogram for $L=240$.

Fig. 9 : LG56 model, phase boundaries on the $T-\mu$ plane as been obtained from the TM data ( $N_{x}=12$ : dashed lines; $N_{x}=18$ : open dots and continuous lines) and from exact low-temperature expansions (full squares). Above: Solid-liquid coexistence (from left to right, squares refer to $\delta=0,-0.01,-0.02,-0.05,-0.1,-0.2)$; below: Liquid-vapor coexistence (from left to right, squares refer to $\delta=0,0.01,0.02,0.05,0.1,0.15$ ). As $\delta$ grows, the truncated expansions become less and less reliable until consistency with TM data is lost.

Fig. 10 : LG56 model, phase boundaries on the $T$-P plane. Data and notation as in Fig. 9.

Fig. 11 : LG34 model, phase boundaries on the $T$ - $\mu$ plane as been obtained from the TM data $\left(N_{x}=14\right.$ : open dots and continuous lines) and from exact low-temperature expansions (full squares). Above: Solid-liquid coexistence (from left to right, squares refer to $\delta=0,-0.01,-0.02,-0.05,-0.1,-0.2)$; below: Coexistence between liquid and vapor (from left to right, squares refer to $\delta=0,0.01,0.02,0.05,0.1,0.2,0.5$ ). As $\delta$ grows, the truncated expansions become less and less reliable until consistency with TM data is lost.

Fig. 12 : LG34 model, phase boundaries on the $T$-P plane. Data and notation as in Fig. 11. 
TABLE I:

\begin{tabular}{|r|r|r|r|r|}
\hline model & $N_{x}$ & $\mathcal{N}_{\text {NLU }}$ & $M_{1}$ & $M_{2}$ \\
\hline \hline LG34 & 14 & 42 & 397357 & 14715 \\
\hline \hline LG56 & 6 & 24 & 88 & 16 \\
\hline LG56 & 12 & 48 & 7768 & 385 \\
\hline LG56 & 18 & 72 & 686905 & 19599 \\
\hline
\end{tabular}




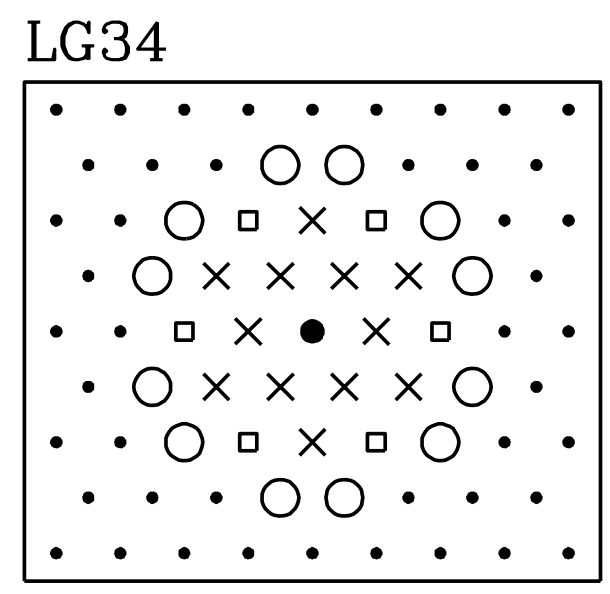

LG56

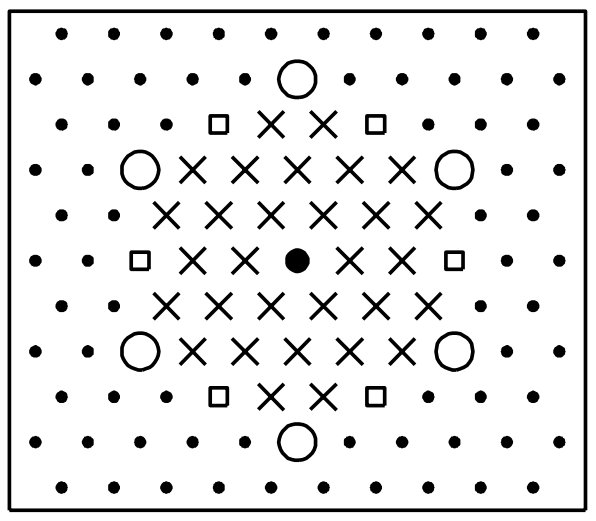

FIG. 1: Pictorial description of the lattice-gas models defined in Eq. (2.1). When a particle is placed in the position marked as a full dot, crosses denote forbidden sites for the centers of other particles. Open squares denote the lattice sites that are occupied by the nearest-neighbor particles of the central particle in the close-packed solid A. Open dots denote attractive sites for the central particle: Here are placed its nearest-neighbor particles in the loose-packed solid B. In the LG34 model, these attractive sites are twelve, contributing a twofold degeneracy to the spatial orientation of solid B. 


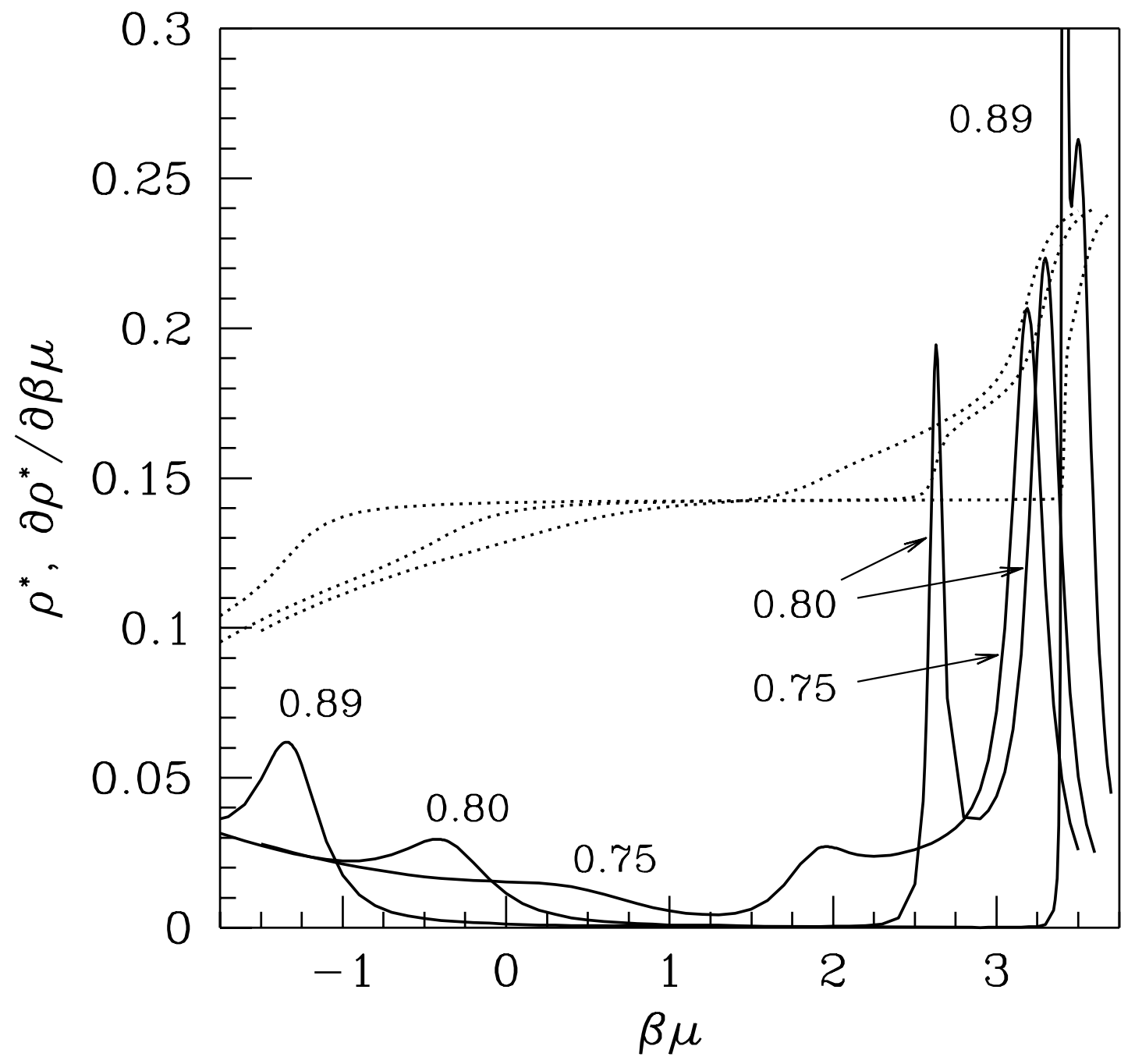

FIG. 2: LG34 model, TM results for $N_{x}=14$. The reduced number density (dotted lines) and its $\beta \mu$ derivative (continuous lines) are shown for three isotherms, $\beta \epsilon=0.75,0.80$, and 0.89 . To help the eye, straight lines are drawn between data points. Peaks in the density derivative are the imprint of phase boundaries: As a rule, the more pronounced a maximum is, the stronger the phase transition. The confluence of three distinct phases (solid A, solid B, and liquid) at $\beta \epsilon \approx 0.90$ is quite transparent from the behavior at $\beta \epsilon=0.89$ (see also Fig. 3 inset). Upon raising the temperature, the region of solid B gradually shrinks until its boundaries fade away. However, in the thermodynamic limit, there will reasonably be no path to go smoothly from fluid to solid B. 


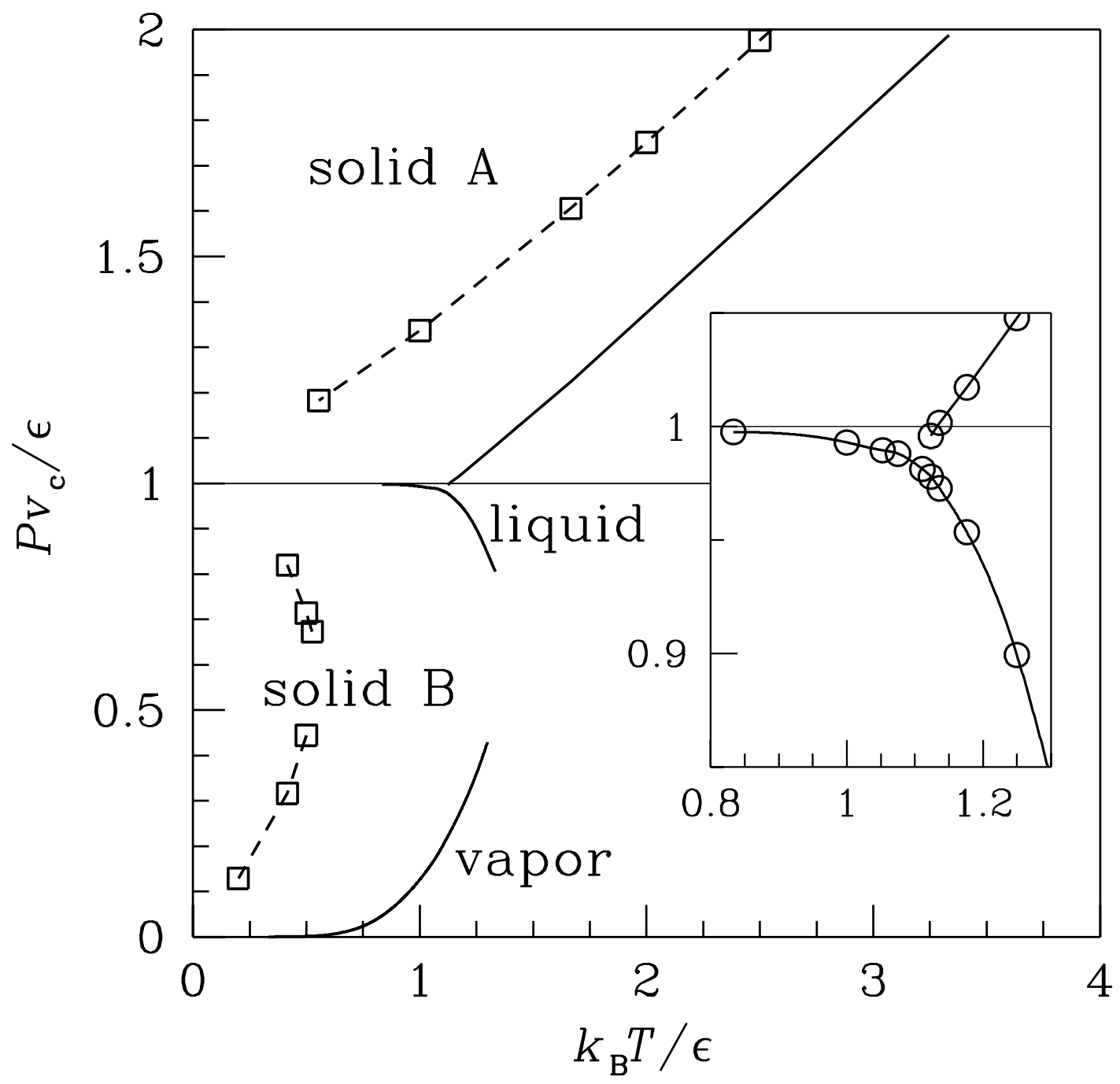

FIG. 3: LG34 model, TM results for $N_{x}=14$. Overall phase diagram of the system as resulting from joining the $(T, P)$ points that correspond to $\partial \rho^{*} / \partial \beta \mu$ peaks. The LG34 potential (open dots and continuous lines) is contrasted with the LG3 repulsive law of Ref. [16] ( $N_{x}=14$, open squares and dashed lines). Spline interpolants drawn between LG34 data points mark first-order phase boundaries; the interruptions are finite-size effects. The fluid phase is vapor-like at low pressure and liquid-like at high pressure. While both models can exist in three phases (solid A, solid B, and fluid), LG34 is peculiar in that it shows a solid-solid transition with volume contraction on heating at constant pressure (inset). 


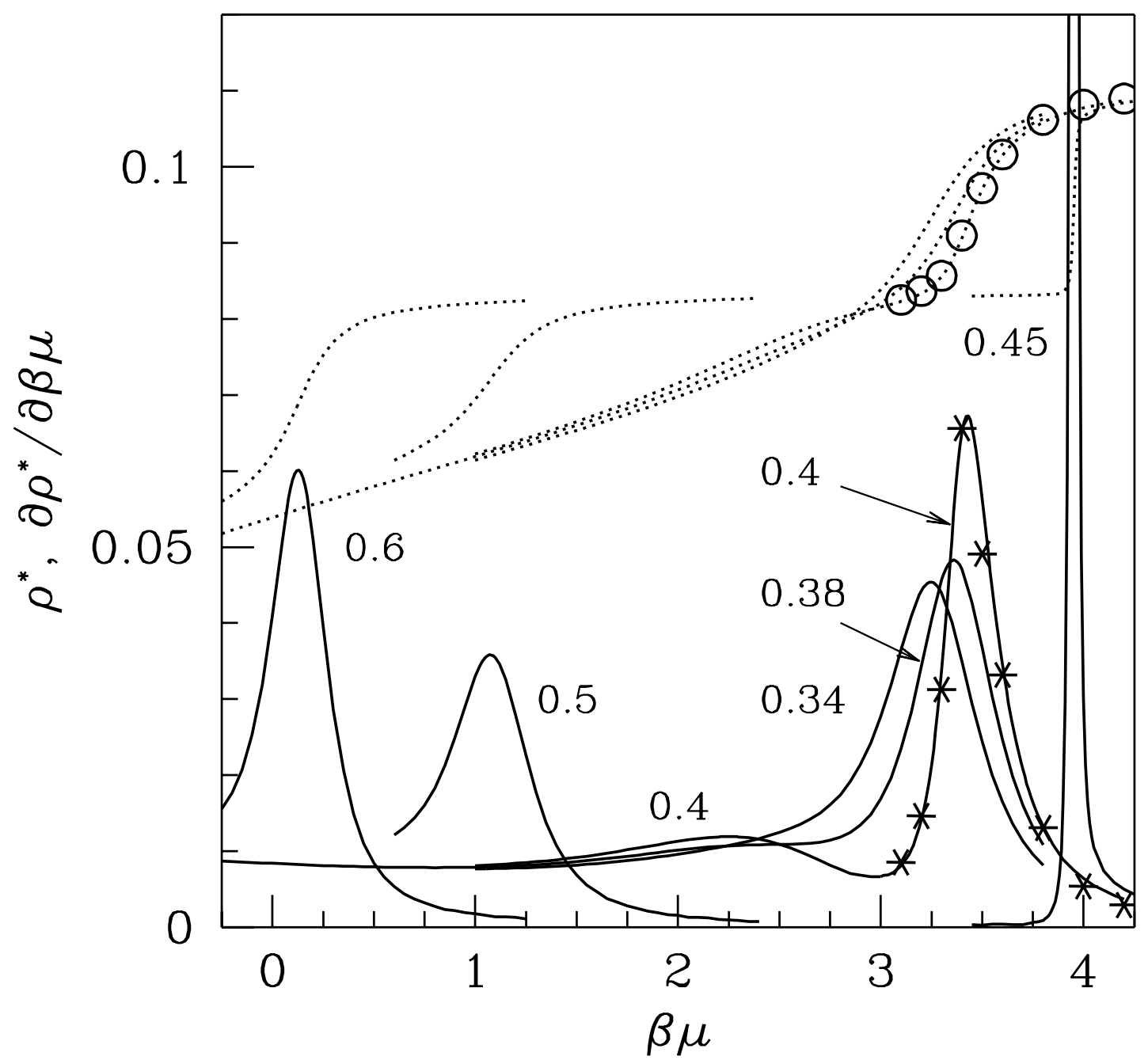

FIG. 4: LG56 model, TM results for $N_{x}=18$. The reduced number density (dotted lines) and its $\beta \mu$ derivative (continuous lines) are shown for a number of isotherms, $\beta \epsilon=0.34,0.38,0.4,0.45,0.5,0.6$, and 0.7. To help the eye, straight lines are drawn between data points. MC data for a $18 \times 360$ periodic sample, relative to $\beta \epsilon=0.4$, are also shown: Reduced number density (open dots) and its $\beta \mu$ derivative (asterisks), as computed through the number fluctuations - see Eq. (3.2). Upon increasing the temperature, the liquid-vapor peak progressively broadens till it disappears at $\beta \epsilon=$ 0.38. The solid-liquid peak is present at all temperatures: It is already very sharp for $\beta \epsilon=0.45$, becoming even sharper for lower temperatures (not shown). 


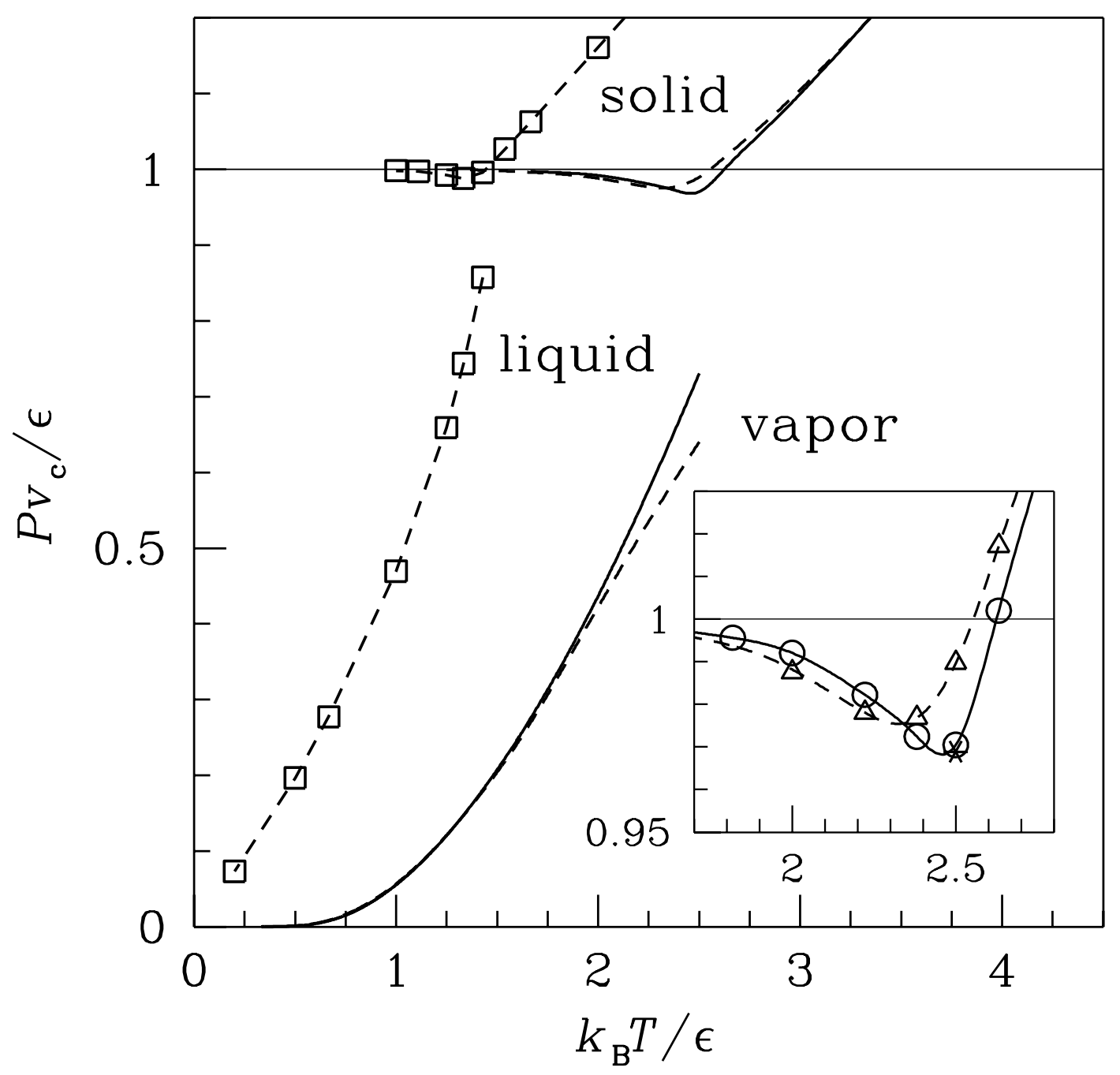

FIG. 5: LG56 model, TM phase diagram for $N_{x}=12$ (open triangles and dashed lines) and 18 (open dots and continuous lines). The phase diagram of LG56 is contrasted with that of LG5 ( $N_{x}=18$, open squares and dashed lines). Both models can exist in three phases: However, while the dense phase of low pressure is solid B for the purely-repulsive LG5 model, the analogous phase for LG56 is liquid, at least for high temperatures (see my arguments in Section 4.B). The LG56 model is peculiar in that an inverse melting occurs (inset). The asterisk is a point on the solidliquid coexistence line of the wider LG56 strip that was constructed by MC (see main text). The shallow minimum in the solid-solid $P_{\operatorname{cox}}(T)$ of LG5 is probably a finite-size effect since this feature is less evident for $N_{x}=18$ than for $N_{x}=12$ (not shown). 

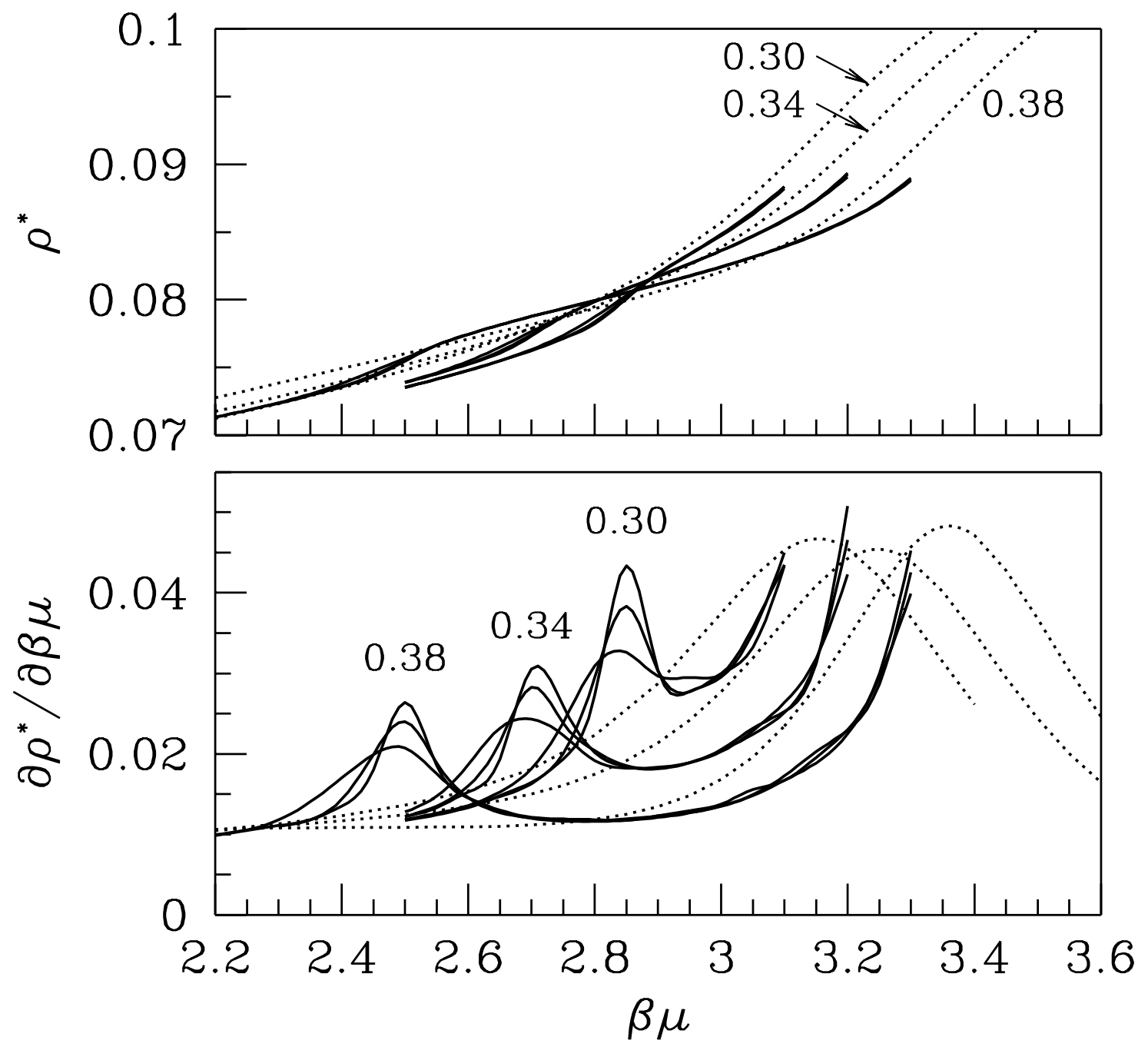

FIG. 6: LG56 model, data for three distinct isotherms, $\beta=0.30,0.34$, and 0.38. Above: reduced number density $\rho^{*}$; below: $\beta \mu$ derivative of $\rho^{*}$. TM data for $N_{x}=18$ (dotted lines) are contrasted with spline interpolants of the MC results for $L \times L$ lattices, with $L=120,180$, and 240 (continuous lines). The MC density derivative is computed through the number fluctuations via Eq. (3.2) (the larger $L$, the more pronounced the $\partial \rho^{*} / \partial \beta \mu$ maximum is). While distinctly recording the freezing transition at all temperatures, a strip of 18 sites is not large enough to follow the boundary between vapor and liquid beyond a reduced temperature of $1 / 0.38$. On the basis of the present MC evidence, it is hard to say whether the liquid-vapor boundary survives at all temperatures or it rather turns into a non-critical disorder line at a finite temperature (the scaling of the MC compressibility at the estimated transition point is inconclusive). 


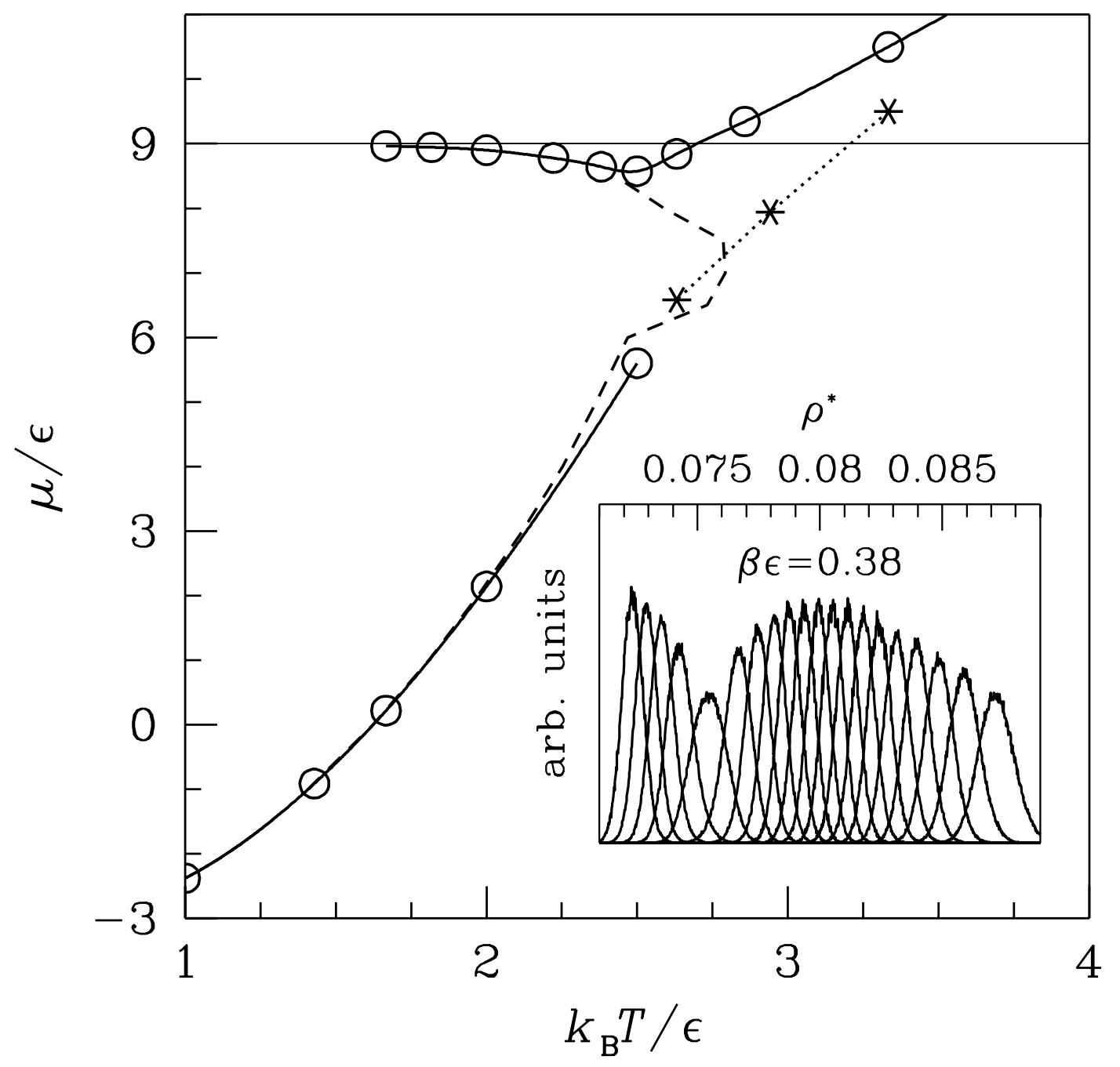

FIG. 7: LG56 model, phase diagram on the $T$ - $\mu$ plane: TM results for $N_{x}=18$ (open dots and continuous lines) plus three MC data points for a $240 \times 240$ lattice (asterisks), corresponding to the location of the $\partial \rho^{*} / \partial \beta \mu$ maxima in Fig. 6. A further dashed line connects TM data points for $N_{x}=18$ recording maxima of $\partial \rho^{*} / \partial \beta \mu$ along constant- $\mu$ cuts. Inset: $\beta \mu$ evolution (from left to right) in the range from 2.30 to 3.25 , with steps of 0.05 , of the density histogram for $\beta=0.38$ and $L=240$. It appears from the inset that the liquid-vapor transition either turns continuous at high temperatures or it becomes a crossover. 


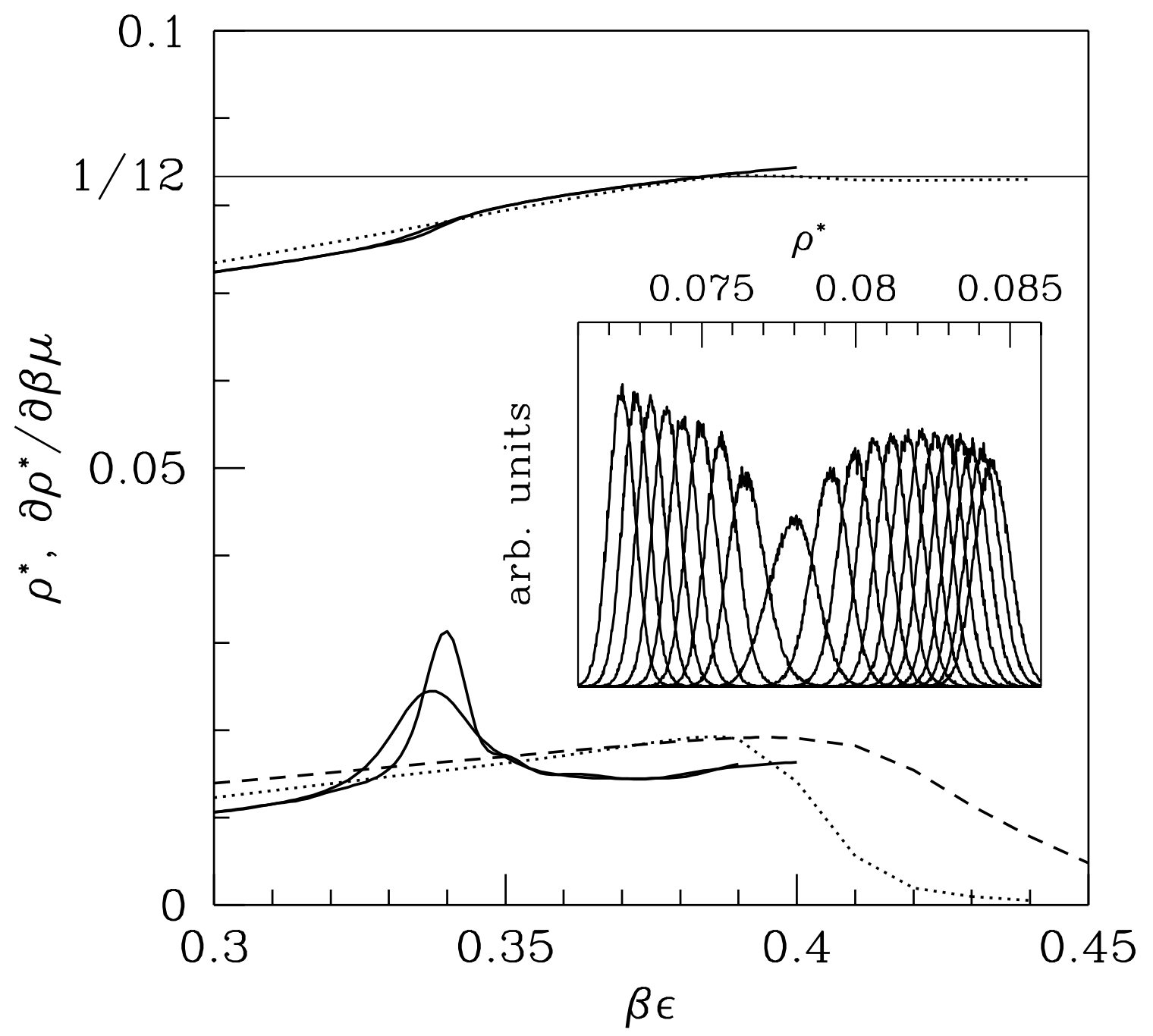

FIG. 8: LG56 model, TM and MC data for the reduced number density $\rho^{*}$ and its $\beta \mu$ derivative along the $\mu=8 \epsilon$ line. TM data for $N_{x}=12$ (dashed line) and $N_{x}=18$ (dotted lines) are contrasted with MC results for $L \times L$ lattices, with $L=120$ and 240 (continuous lines). The transition at $\beta \epsilon \simeq 0.34$ is from vapor to liquid while a defected solid B structure abruptly appeared when pushing the simulation beyond $\beta \epsilon=0.40$. Inset: $\beta \mu$ evolution (from left to right) in the range from 0.3 to 0.4, with steps of 0.005 , of the density histogram for $L=240$. 

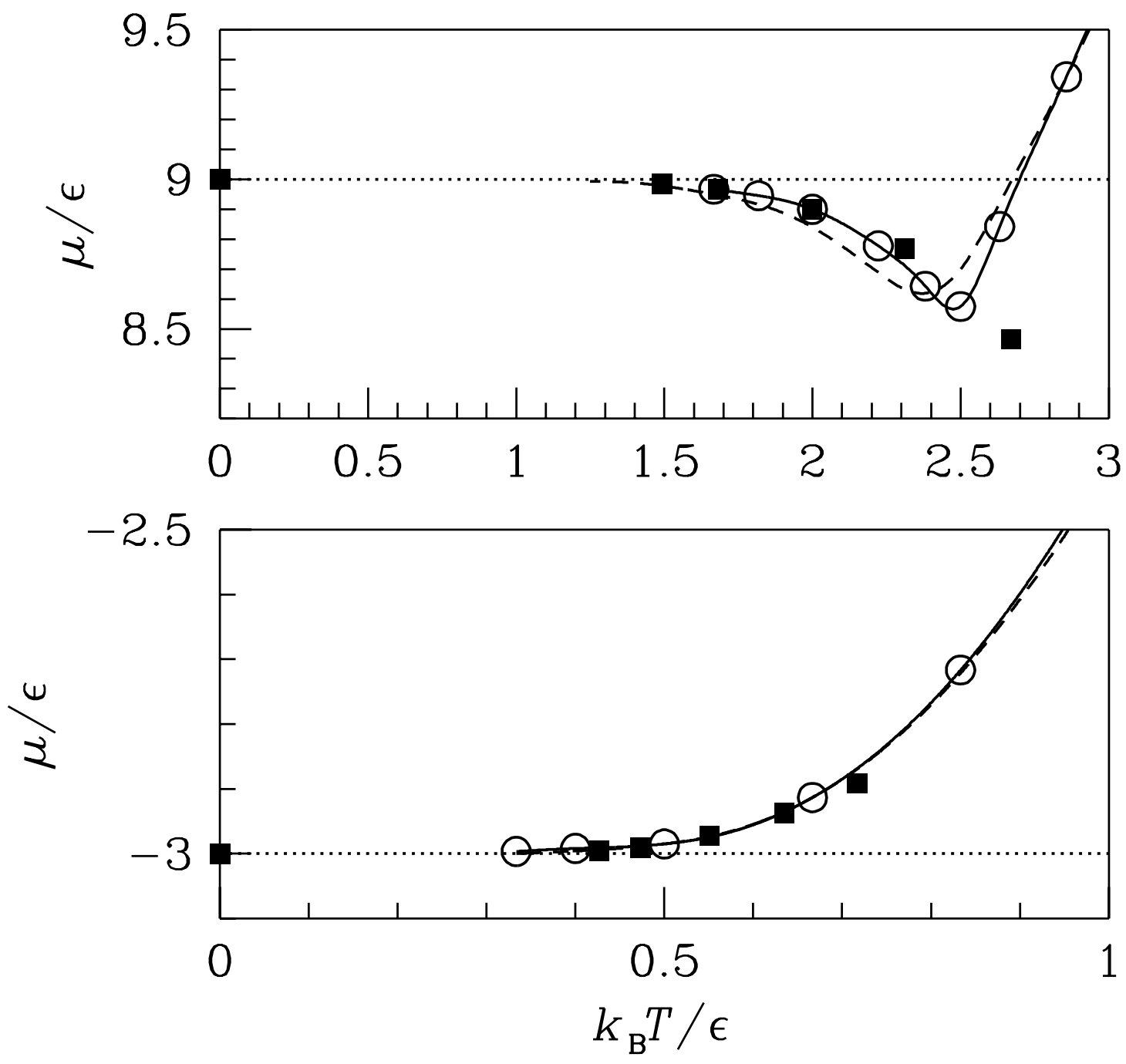

FIG. 9: LG56 model, phase boundaries on the $T$ - $\mu$ plane as been obtained from the TM data ( $N_{x}=12$ : dashed lines; $N_{x}=18$ : open dots and continuous lines) and from exact low-temperature expansions (full squares). Above: Solid-liquid coexistence (from left to right, squares refer to $\delta=0,-0.01,-0.02,-0.05,-0.1,-0.2)$; below: Liquid-vapor coexistence (from left to right, squares refer to $\delta=0,0.01,0.02,0.05,0.1,0.15)$. As $\delta$ grows, the truncated expansions become less and less reliable until consistency with TM data is lost. 

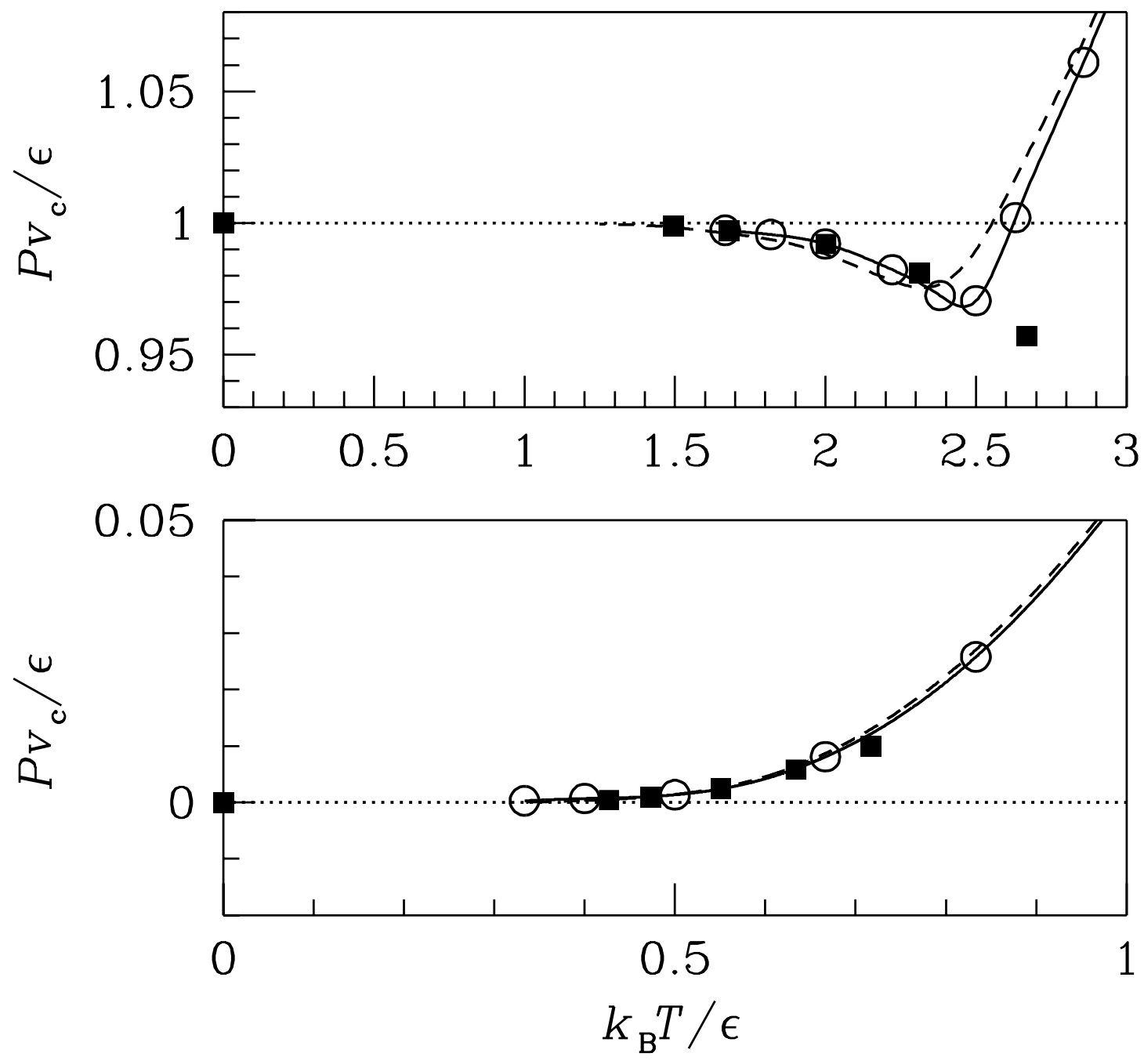

FIG. 10: LG56 model, phase boundaries on the T-P plane. Data and notation as in Fig. 9. 

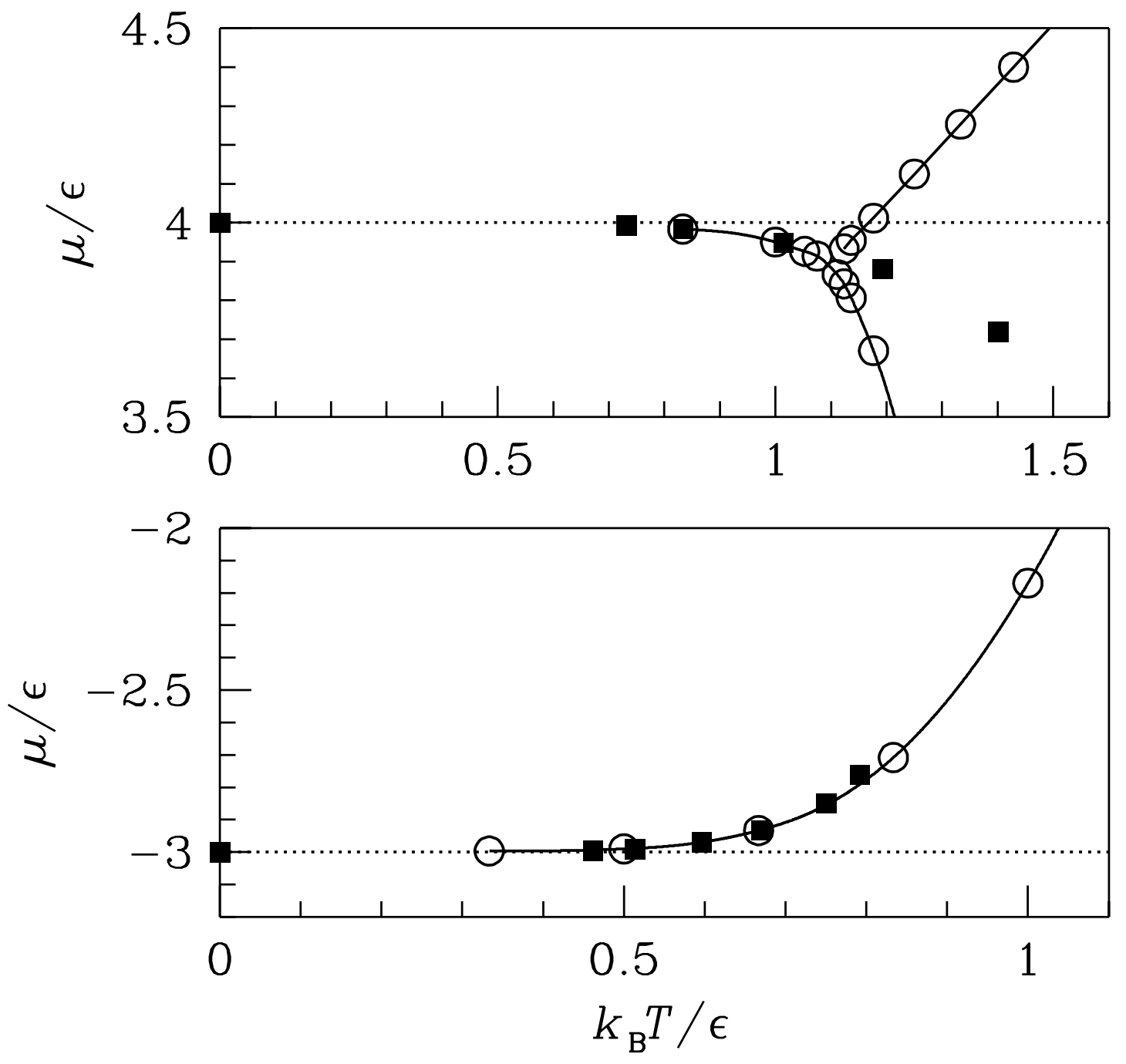

FIG. 11: LG34 model, phase boundaries on the $T$ - $\mu$ plane as been obtained from the TM data $\left(N_{x}=14\right.$ : open dots and continuous lines) and from exact low-temperature expansions (full squares). Above: Solid-liquid coexistence (from left to right, squares refer to $\delta=$ $0,-0.01,-0.02,-0.05,-0.1,-0.2)$; below: Coexistence between liquid and vapor (from left to right, squares refer to $\delta=0,0.01,0.02,0.05,0.1,0.2,0.5)$. As $\delta$ grows, the truncated expansions become less and less reliable until consistency with TM data is lost. 

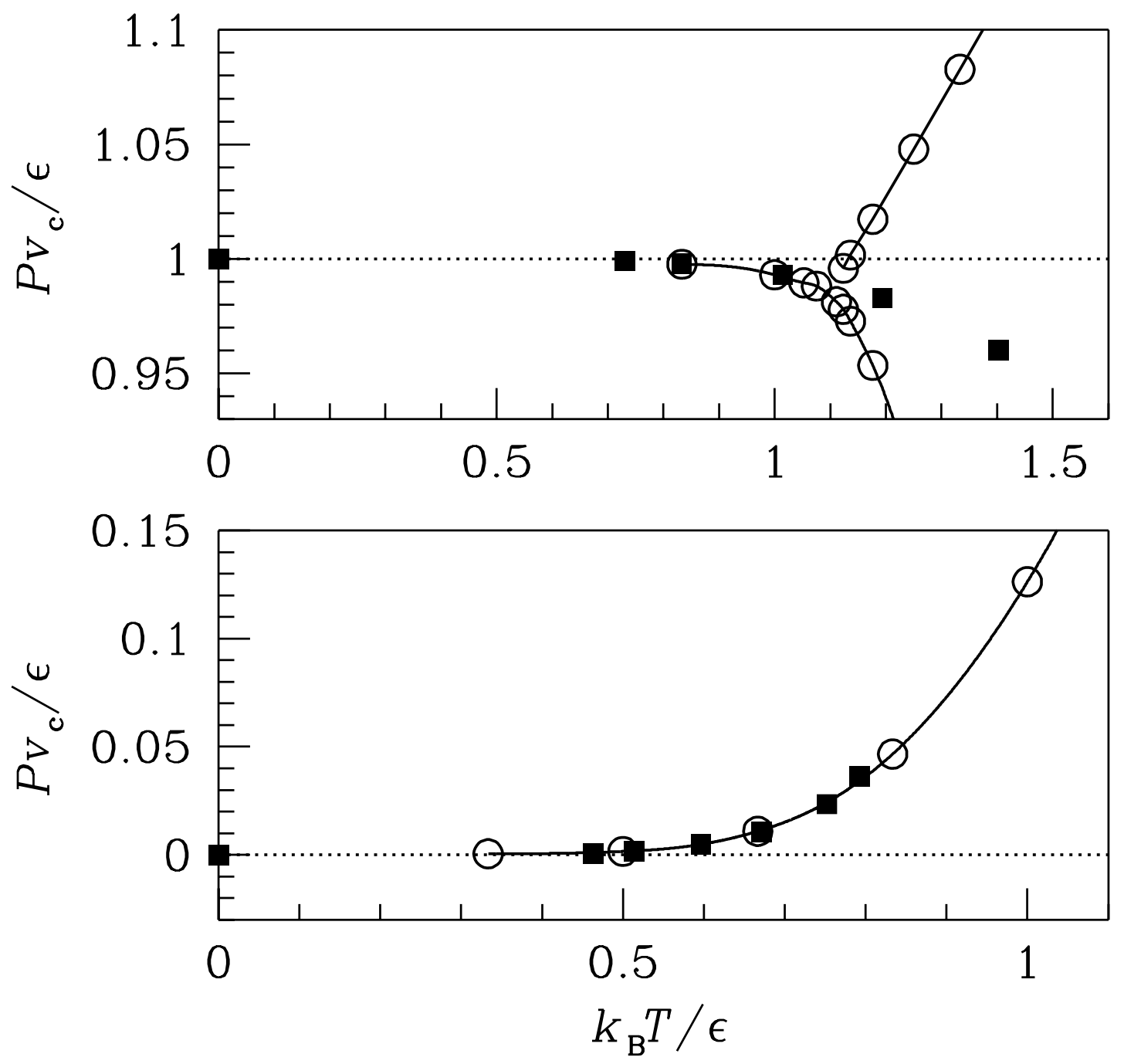

FIG. 12: LG34 model, phase boundaries on the $T$ - $P$ plane. Data and notation as in Fig. 11. 